\title{
Perspectives for positron emission tomography with RPCs
}

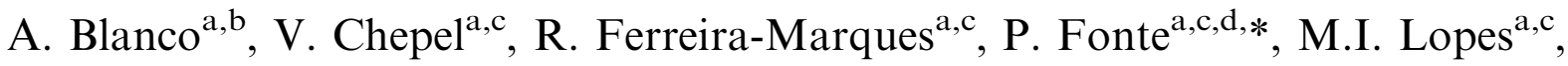 \\ V. Peskov ${ }^{\mathrm{e}}$, A. Policarpo ${ }^{\mathrm{a}, \mathrm{c}}$ \\ ${ }^{\text {a } L I P-L a b o r a t o ́ r i o ~ d e ~ I n s t r u m e n t a c ̧ a ̃ o ~ e ~ F i ́ s i c a ~ E x p e r i m e n t a l ~ d e ~ P a r t i ́ c u l a s, ~ P o r t u g a l ~}$ \\ ${ }^{\mathrm{b}}$ GENP, Dept. Fisica de Particulas, Univ. Santiago de Compostela, Spain \\ ${ }^{\mathrm{c}}$ Departamento de Física da Universidade de Coimbra, Coimbra, Portugal \\ ${ }^{\mathrm{d}}$ Instituto Superior de Engenharia de Coimbra, Coimbra, Portugal \\ ${ }^{\mathrm{e}}$ Royal Institute of Technology, Stockholm, Sweden
}

\begin{abstract}
In this study, we address the feasibility and main properties of a positron emission tomograph (PET) based on RPCs. The concept, making use of the converter-plate principle, takes advantage of the intrinsic layered structure of RPCs and its simple and economic construction. The extremely good time and position resolutions of RPCs also allow the TOFPET imaging technique to be considered.

Monte-Carlo simulations, supported by experimental data, are presented and the main advantages and drawbacks for applications of potential interest are discussed.
\end{abstract}

(C) 2003 Elsevier Science B.V. All rights reserved.

PACS: $29.40 . \mathrm{Cs}$

Keywords: Gaseous detectors; RPC; PET; TOF

\section{Introduction}

Positron Emission Tomography (PET) is a radiotracer imaging technique in which tracer compounds labelled with positron emitting radionuclides are injected into the object of study. These tracer compounds can then be used to track biomedical and physiological processes, with

\footnotetext{
Dresented at "RPC2001-VI Workshop on Resistive Plate Chambers and Related Detectors", Coimbra, Portugal, 26-27 November 2001.

*Corresponding author. Departamento de Física da Universidade de Coimbra, Coimbra 3004-516, Portugal. Tel.: +351-239-833465; fax: +351-239-822358.

E-mail address: fonte@lipc.fis.uc.pt (P. Fonte).
}

applications ranging from the early detection of cancer to neurophysiology studies.

After a short path length, the positron annihilates with an electron of the medium emitting simultaneously two almost anti-parallel $511 \mathrm{keV}$ photons. The coincident detection of both photons identifies the occurrence of an annihilation along the chord joining the detection points and the accumulation of such data allows the reconstruction of the activity distribution in the tissues.

If, additionally, the coincidence time difference (difference in flight time between the pair of photons) is measured (TOF-PET) with an FWHM accuracy $\Delta t$, the position of the annihilation along the chord may be identified with an FWHM 
accuracy $\Delta L$ given by

$\Delta L=c \Delta t / 2=c\left(2.36 \sqrt{2} \sigma_{t}\right) / 2$

where $\sigma_{t}$ is the rms time accuracy per photon. The numerical factors combine to yield

$\Delta L[\mathrm{~mm}] \approx 0.15 \Delta t[\mathrm{ps}] \approx \sigma_{t}[\mathrm{ps}] / 2$.

In any foreseeable system, the localisation accuracy given by Eq. (2) is much coarser than the desired image granularity (few $\mathrm{mm}$ ) and therefore the image reconstruction procedure cannot be avoided. However, by including the TOF information, along with other advantages [1], one gains a sensitivity improvement of the order of $L / \Delta L$, where $L$ is the typical object length, thus reducing the number of events needed for the image reconstruction [2]. For this reason the investigation of TOF-PET systems is being actively pursued (e.g. Refs. [3,4]).

In order to compare a TOF-PET system with a conventional (non-TOF) one and account for the above-mentioned increase in sensitivity when using TOF information, one may introduce an intrinsic sensitivity merit factor defined as

$f \approx\left(\frac{\varepsilon_{\mathrm{TOF}}}{\varepsilon}\right)^{2} \frac{L[\mathrm{~mm}]}{0.15 \Delta t[\mathrm{ps}]}$

where $\varepsilon_{\mathrm{TOF}}$ and $\varepsilon$ are the quantum efficiencies for a single gamma photon in TOF and non-TOF systems, respectively.

However, in a practical PET tomograph many additional factors determine the overall system sensitivity in terms of dose given to the patient for an exam requiring a certain image quality (see for instance Refs. [5,6] and references therein). Examples of such factors would include:

(1) System price, which largely determines the affordable field of view (FOV: axial detector length);

(2) Localisation accuracy of the photon interaction points, which includes the depth-ofinteraction (DOI) information, the position resolution along the axial and transaxial directions of the tomograph and the errors due to Compton scattering in the detector;

(3) The count rate capability, including detector occupancy and data acquisition (DAQ) throughput;
(4) The time resolution of the counter and of the DAQ, which influences the number of random coincidences;

(5) Counter geometry, in particular the use (2DPET) or not (3D-PET) of collimating septa dividing the FOV in nearly independent rings.

For most of these performance factors, there will be considerable differences between the RPC TOF-PET and the crystal PET approaches. However, a full discussion of such aspects and of their interplay cannot be done in the present report, being only outlined the most prominent differences to be expected between these approaches based on simulations and on experimental results.

\section{The RPC TOF-PET concept}

The RPC TOF-PET concept is based on the converter-plate principle [7] and takes advantage of the naturally layered structure of RPCs, of its simple and economic construction, excellent time resolution ( $60 \mathrm{ps} \sigma$ even for single gaps equipped with position-sensitive readout [8,9]) and very good intrinsic position accuracy $(50 \mu \mathrm{m}$ online in digital readout mode [10]).

A possible RPC structure for TOF-PET applications is shown in Fig. 1. The incoming gamma photons will interact with the electrode materials through the photoelectric and Compton processes.

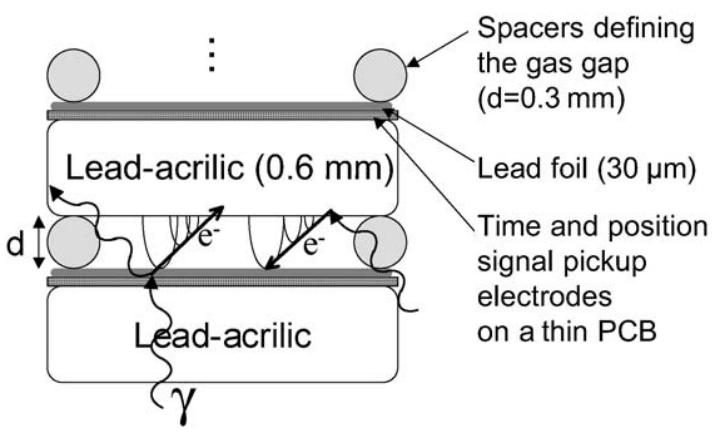

Fig. 1. Counter construction assumed in the GEANT4 simulations presented below with a schematic indication of the most relevant physical processes. Each layer has a total thickness of $1 \mathrm{~mm}$ resulting in a $10 \mathrm{~cm}$ thick 100-layer counter. 
The resulting electrons strongly scatter in the material and eventually emerge into the gas gap, ionising the gas and initiating avalanches along their path. The exponential dependence of the avalanche final charge with the position of the initial charges assures that only those avalanches initiated close to the cathode will be detected. (In Fig. 1, the lead foil was chosen as cathode.)

Other approaches based on the converter-plate principle, although offering a much poorer time resolution, may be found in Refs. [11,12].

\section{Experimental and Monte-Carlo results}

\subsection{Quantum efficiency and energy sensitivity}

Clearly, the weaker point of the converter-plate approach to medium-energy photon detection is the low quantum efficiency (probability of detection per incident photon) relative to inorganic scintillators.

Calculations show that a stack of very thin lead foils obtains the best results. However, for keeping the mechanical accuracy of the counter, the foils must be mounted on a rigid resistive electrode. A possible material may be lead-loaded acrilic (LAused for transparent X-ray protections) which offers its best performance at a convenient thickness of $0.5-1 \mathrm{~mm}$.

We measured a quantum efficiency of 0.0044 for single lead foils in good agreement with Refs. [7,12], while the corresponding GEANT4 simulation yielded a value of 0.0042 , validating GEANT4 for this application.

Further simulations suggest that a 140-layer counter with the structure shown in Fig. 1 should reach an efficiency per photon $\varepsilon_{\mathrm{TOF}}=22 \%$ (Fig. 2), yielding a relative merit factor Eq. (3) for a $24 \mathrm{~cm}$ diameter object

$f \approx\left(\frac{0.2}{0.8}\right)^{2} \frac{240}{0.15 \times 300}=0.33$

where a coincidence time resolution of $300 \mathrm{ps}$ FWHM was used (see Section 3.3).

The proposed system would show a sensitivity handicap by a factor of three when compared with a standard crystal PET, all other variables being

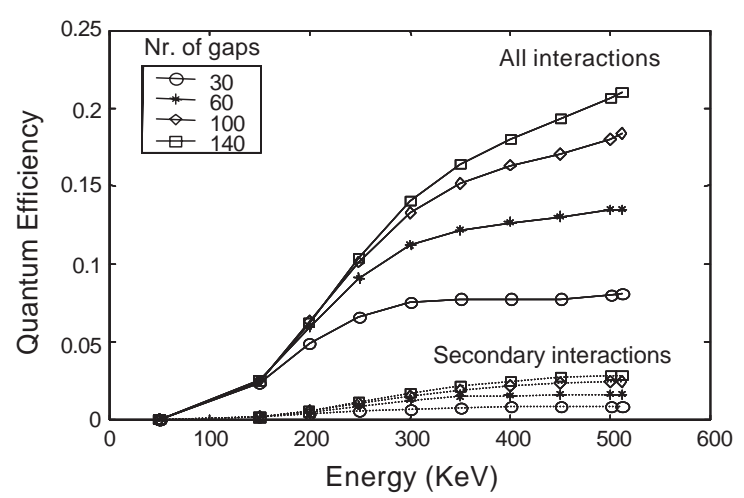

Fig. 2. Simulated (GEANT4) quantum efficiency as a function of the incident photon energy for a stack of counters (see Fig. 1). For a large number of plates the values scale almost linearly with the energy above $\sim 100 \mathrm{keV}$. Only a small fraction of the secondary interactions (from counter-scattered photons) is visible.

the same. However. for some special applications (see Section 3.5) there are advantages in the present proposal that may easily outweigh this handicap.

Using photon energy distribution data generated by the public domain SIMSET simulation package [13], it was determined that the energy sensitivity feature of the present approach is equivalent, in terms of rejection of coincidences involving a scattered photon, to an energy discrimination threshold of $300 \mathrm{keV}$.

\subsection{Position resolution}

A strong point of the converter-plate approach is its position resolution capability (e.g. Ref. [14]).

In Fig. 3, we show the GEANT4-simulated image of a disc phantom in water imaged by a 16layer counter with a structure similar to that shown in Fig. 1. The inner radius was $4 \mathrm{~cm}$ and the stack was $16 \mathrm{~mm}$ thick. All high-energy processes were simulated including the positron path range (using the $\beta$ energy distribution from ${ }^{18} \mathrm{~F}$ ) and photon non-colinearity. The FWHM RPC position resolution was assumed to be twice the width of the gas gap $(2 \times 0.3=0.6 \mathrm{~mm})$ for electrons ejected from the anode and $0.2 \mathrm{~mm}$ for electrons ejected from the cathode. The image was reconstructed by the standard algorithm of filtered 


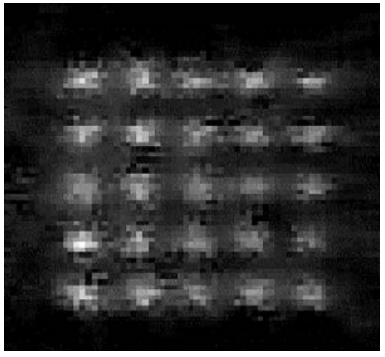

Fig. 3. Monte-Carlo simulation (GEANT4) of $40 \mu \mathrm{m}$ diameter discs spaced by $1 \mathrm{~mm}$. The discs are clearly resolved, in agreement with the corresponding $0.38 \mathrm{~mm}$ FWHM Point Spread Function.

backprojection. The contrast of the resulting image was enhanced by subtraction of the lowfrequency background.

Since the DOI of the photons is millimetrically determined by the depth of the firing gap, no parallax effect was observed in the simulations.

The $40 \mu \mathrm{m}$ diameter discs spaced by $1 \mathrm{~mm}$ are clearly resolved. The corresponding Point Spread Function was determined by reconstructing point sources and shows a width of $0.38 \mathrm{~mm}$ FWHM.

\subsection{Time resolution}

Measurements taken with $0.3 \mathrm{~mm}$ single-gap RPCs made with electrodes of glass and aluminium yielded an FWHM coincidence time resolution $\Delta t=300 \mathrm{ps}$, equivalent to $\sigma_{t}=90 \mathrm{ps}$, for $511 \mathrm{keV}$ photon pairs.

Since such counters are known to deliver a resolution $\sigma_{t} \approx 60 \mathrm{ps}$ [8] when irradiated with minimum ionising particles and no optimisation of the mechanical and operational parameters for this application was done yet, further improvements are reasonably to be expected.

Anyhow, these results already compare favourably with the typical TOF-PET coincidence resolution of $500 \mathrm{ps}$ FWHM achievable with $\mathrm{BaF}_{2}$ or LSO segmented crystals [3] and even with large $\mathrm{BaF}_{2}$ crystals yielding 340 ps FWHM [15].

\subsection{Rate capability}

Since glass (or even bakelite) RPCs are amongst the gas counters with lower rate capability, it

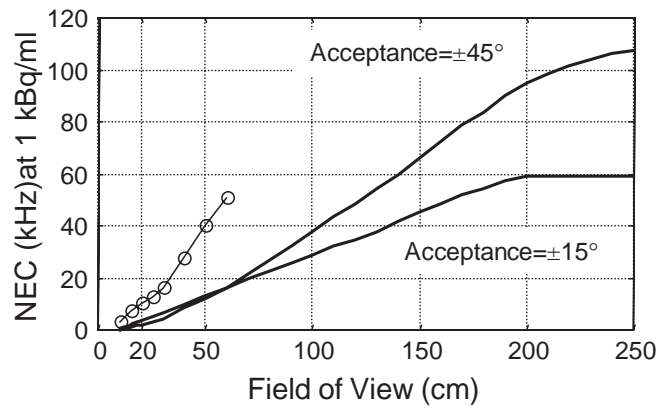

Fig. 4. Simulated NEC rates as a function of the axial FOV. The circles correspond to standard 3D PET [17] and the thick lines to RPC TOF-PET. A sensitivity increase by a factor of 10 over standard 3D PET $(20 \mathrm{~cm}$ FOV) may be possible for wholebody examinations.

should be asked whether these counters might withstand the very large counting rates desirable for PET.

The counting rate capability of timing glass RPCs under continuous irradiation is around $200 \mathrm{~Hz} / \mathrm{cm}^{2}$ [16], corresponding to $20 \mathrm{kHz} / \mathrm{cm}^{2}$ for a 100-layer counter. For the large-area application considered in the next section, the corresponding maximum singles count rate would be $1.2 \mathrm{GHz}$, which is likely unreachable due to other reasons such as random coincidences and DAQ throughput limitations.

Therefore, it seems that for large-area counters the maximum system counting rate will not be limited by the RPCs rate capability.

\subsection{Field of view and overall system sensitivity}

The present approach may allow the realisation of affordable full-body FOV scanners.

Simulations by other authors [17] suggest that increasing the axial FOV of a standard crystal PET from 20 to $60 \mathrm{~cm}$ would improve the noiseequivalent count rate $\left(\mathrm{NEC}^{1}[18]\right)$ by a factor of five to eight. Such results are shown in Fig. 4 along with our own simulations based on the SIMSET package [13].

\footnotetext{
${ }^{1}$ The number of true coincidences that would create an image of similar quality in the absence of noise (scattered and random coincidences).
} 
Our curves correspond to a 801 water cylinder with a diameter of $24 \mathrm{~cm}$ and $1.8 \mathrm{~m}$ length. The simulation is meant to determine only geometrical effects and does not include rate-dependent effects like system dead time or random coincidences, while the results of Ref. [17] were obtained with the Zubal phantom, using larger counting rates and modelling rate-dependent effects. To allow a comparison between both results, the curve from Ref. [17] was normalised to an NEC sensitivity of $10 \mathrm{kHz} /(\mathrm{kBq} / \mathrm{ml}$ ) for a $20 \mathrm{~cm}$ FOV (a common value for 3D-PET: e.g. Ref. [19]) and our curve to $3.3 \mathrm{kHz} /(\mathrm{kBq} / \mathrm{ml})$ following Eq. (4). Two acceptance angles ${ }^{2}$ were considered.

The results suggest that a $2.5 \mathrm{~m}$ FOV RPC TOFPET would show an NEC sensitivity improvement by a factor 10 over the standard PET sensitivity, vastly outweighing the intrinsic sensitivity handicap for whole-body examinations.

\section{Conclusions}

We assessed several key features of an economic and positionally accurate RPC-based approach to the TOF-PET medical imaging technique.

Efficiency up to $22 \%$ for $511 \mathrm{keV}$ photons may be achievable for a 140-layer counter along with an energy sensitivity equivalent in terms of scatter rejection to an energy cutoff at $300 \mathrm{keV}$.

A coincidence time resolution of 300 ps FWHM was measured for $511 \mathrm{keV}$ photons, comparing favourably with the values achievable with segmented fast crystals.

The excellent $(380 \mu \mathrm{m}$ FWHM $)$ parallax-free position resolution expected for the reconstructed images (from small diameter counters) may have direct interest for the high-resolution imaging of small animals required by pharmaceutical research.

The simple structure of RPC-type detectors and the consequent possibility of economic construction in large areas opens the way to consider also whole-body field-of-view TOF-PET counters. Simulations suggest that such systems may reach an

\footnotetext{
${ }^{2}$ The maximum angle of the incoming photons relative to the radial direction.
}

overall sensitivity one order of magnitude larger than present day crystal-based PET scanners. Such improvements may open new medical applications by lowering considerably the radiation hazard involved in whole-body PET examinations and simultaneously increase the patient throughput.

\section{Acknowledgements}

This study was supported by the FCT project CERN/P/FIS/40111/2000.

The authors gratefully acknowledge the "Centro de Supercomputación de Galicia" (CESGA) for providing very substantial computational resources and the very special collaboration of Dr. Carmen C. Bueno.

\section{References}

[1] S.E. Derenzo, et al., Critical instrumentation issues for $<2 \mathrm{~mm}$ resolution high sensitivity brain PET, in: $\mathrm{K}$. Uemura, N.A. Lassen, T. Jones, I. Kanno (Eds.), Quantification of Brain Function: Tracer Kinetics and Image Analysis in Brain PET, Elsevier, Amsterdam, 1993, pp. 25-37 (http://cfillbl.gov/instrumentation/Pubs/QuantBrainFunc.pdf).

[2] T.F. Budinger, J. Nucl. Med. 24 (1983) 73.

[3] W.W. Moses, S.E. Derenzo, IEEE Trans. Nucl. Sci. NS-46 (1999) 474.

[4] T. Yamaya, et al., Phys. Med. Biol. 45 (2000) 3125.

[5] W.W. Moses, Nucl. Instr. and Meth. A 471 (2001) 209.

[6] D. Crosetto, A modular VME or IBM PC based data acquisition system for multi-modality PET/CT scanners of different sizes and detector types, Presented at the IEEE Nuclear Science Symposium and Medical Imaging Conference, Lyon, France, 2000, Published in the Conference Record, http://www.3d-computing.com/pb/ieee2000563. pdf.

[7] J.E. Bateman, Nucl. Instr. and Meth 221 (1981) 131.

[8] A. Blanco, et al., Nucl. Instr. and Meth. A 478 (2002) 170.

[9] A. Blanco, et al., Single-gap timing RPCs with bidimensional position sensitive readout for very accurate TOF systems, Nucl. Instr. and Meth. A, these proceedings.

[10] V. Peskov, P. Fonte, Gain, rate and position resolution limits of micropattern gaseous detectors, Presented at the PSD99-Fifth International Conference on Position-Sensitive Detectors, London, England, 1999, Also preprint LIP/ 01-06 (http://xxx. lanl. gov/abs/physics/0106017).

[11] A. Jeavons, et al., IEEE Trans. Nucl. Sci. NS-30 (1983) 640.

[12] J. Lacy, et al., Nucl. Instr. and Meth. A 471 (2001) 88. 
[13] T.K. Lewellen, et al., The SimSET program, in: M. Ljungberg, S.-E. Strand, M.A. King (Eds.), Monte Carlo Calculations in Nuclear Medicine: Applications in Diagnostic Imaging, Institute of Physics, Philadelphia, 1998, pp. $77-92$.

[14] A. Jeavons, et al., IEEE Trans. Nucl. Sci. NS-46 (3) (1999) 468.

[15] J. Cederk, et al., Nucl. Instr. and Meth. A 471 (2001) 200.
[16] A. Akindinov, et al., Nucl. Instr. and Meth. A 456 (2000) 16.

[17] R.D. Badawi, et al., IEEE Trans. Nucl. Sci. NS-47 (2000) 1228.

[18] S.C. Strother, et al., IEEE Trans. Nucl. Sci. NS-37 (1990) 783.

[19] Lars-Eric Adam, et al., J. Nucl. Med. 42(12) (2001) 1821. 